\title{
Glucocorticoids revealed to augment intestinal epithelial barrier function
}

Glucocorticoids are known to restore barrier function in patients with IBD; however, it is not known whether this is a result of attenuated inflammation, or if the therapy has an epithelial-specific effect. A team from Germany has now shed light on this question.

The researchers used Caco- 2 cells as an in vitro model of the intestinal epithelium. "By culturing these cells on semipermeable filter supports, they were allowed to polarize and differentiate into cells resembling mature small intestinal

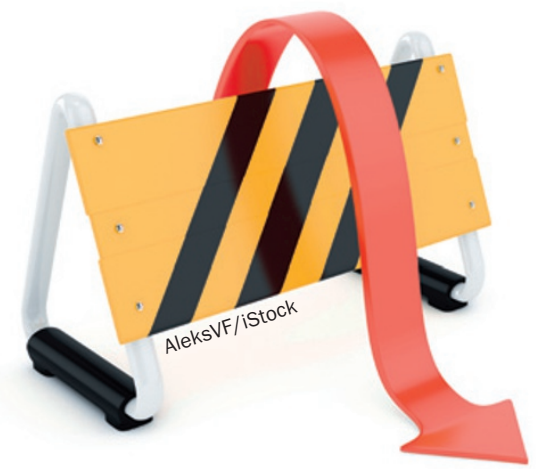

enterocytes," explains lead author Andreas Fischer (Charité Universitätsmedizin Berlin, Germany). This method also enabled the team to assess tight junction permeability, in addition to the size and charge selectivity of the paracellular pathway, by measuring transepithelial resistance. They found that administration of glucocorticoids resulted in a timedependent and dose-dependent increase in transepithelial electrical resistance and reduced paracellular cation flux.

Fischer and colleagues also assessed the expression levels of key components of the tight junctions. The expression of claudin 2 (which is involved in pore formation) was reduced, whereas expression of claudin 4 (which is involved in sealing tight junctions) was upregulated. "We also found that reduced ion permeability and changes in the claudin expression pattern depended on glucocorticoids upregulating the expression of MKP-1, a phosphatase implicated in the deactivation of MAPK signalling," says Fischer.

The authors conclude that their results suggest that barrier augmentation could be a previously unrecognized mechanism that might contribute to the therapeutic efficacy of glucocorticoids in patients with IBD. However, they note that it is not clear from their study which of the downstream targets of MKP-1 are involved in regulating intestinal tight junction permeability. "Elucidating these pathways could enable the development of strategies aimed at preserving the local protective functions of glucocorticoids while avoiding their detrimental systemic adverse effects," says Fischer.

\section{Claire Greenhill}

Original article Fischer, A. et al. Glucocorticoids regulate barrier function and claudin expression in intestinal epithelial cells via MKP-1. Am. J. Physiol. Gastrointest. Liver Physiol. doi:10.1152/ajpgi.00095.2013 